\title{
Two-photon-induced birefringence in azo-dye bearing polyimide; the birefringence changes versus the writing power
}

\author{
A. Kozanecka-Szmigiel • K. Switkowski • \\ E. Schab-Balcerzak • E. Grabiec
}

Received: 25 February 2011 / Revised version: 30 March 2011 / Published online: 10 May 2011

(C) The Author(s) 2011. This article is published with open access at Springerlink.com

\begin{abstract}
Ultra-short high-intensity light pulses were utilized to induce the optical birefringence in a polyimide material possessing the azo-dye covalently bonded to the main chain. The obtained results showed that a two-photon absorption process was involved in a creation of the sample birefringence which, to the best of our knowledge, was not previously reported for polyimide materials. The growths and decays of birefringence were examined as functions of the pulse intensities. No damage to the material during the illumination process was detected in a wide range of optical powers applied. High birefringence level of the order of 0.005 was measured.
\end{abstract}

\author{
A. Kozanecka-Szmigiel $(\bowtie) \cdot K$. Switkowski \\ Faculty of Physics, Warsaw University of Technology, \\ 75 Koszykowa Str., 00-662 Warsaw, Poland \\ e-mail: annak@if.pw.edu.pl \\ Fax: +48-22-6282171 \\ K. Switkowski \\ e-mail: krzychu1@gmail.com \\ Fax: +48-22-6282171 \\ E. Schab-Balcerzak · E. Grabiec \\ Centre of Polymer and Carbon Materials, \\ Polish Academy of Sciences, 34 M. Curie-Sklodowska Str., \\ 41-819 Zabrze, Poland \\ E. Schab-Balcerzak \\ e-mail: eschab-balcerzak@cmpw-pan.edu.pl \\ Fax: +48-032-2712969 \\ E. Grabiec \\ e-mail: egrabiec@cmpw-pan.edu.pl \\ Fax: +48-032-2712969 \\ E. Schab-Balcerzak \\ Institute of Chemistry, University of Silesia, 9 Szkolna Str., \\ 40-006 Katowice, Poland \\ e-mail: ewa.schab-balcerzak@us.edu.pl \\ Fax: +48-32-2599978
}

\section{Introduction}

The phenomenon of light-induced birefringence in azobenzene-derivative polymers has been the subject of intense studies motivated by interesting future applications in the area of reversible optical storage [1-3]. The phenomenon arises from a reversible structural change of azo chromophores between a stable trans-and a less stable cisisomers. Multiple trans-cis-trans isomerization cycles under the polymer irradiation lead to the chromophore rearrangement; the material becomes birefringent with the axis perpendicular to the electric vector of the irradiating light.

Numerous studies on photoinduced birefringence have been devoted to a molecular reorientation created in polymer films via a linear absorption process of a continuous $(\mathrm{cw})$ beam. It has been shown that the induced birefringence is influenced by many factors referring to not only the azo-dye and polymer chemical structure but also to the experimental conditions [4-6]. The reorientation of azochromophores has been shown to appear under a pulse beam illumination as well. The birefringence level obtained in this case differs from the one achieved during a $\mathrm{cw}$ light irradiation due to local thermal heating by the pulses [7].

Ultra-short high-intensity laser pulses may induce the sample birefringence via a two-photon absorption process. It is a nonlinear optical process involving the simultaneous absorption of two identical photons of half of the energies of a molecule excited level, with a transition probability dependent on the square of the pulse intensity. Unlike the linear absorption, in which the light beam is absorbed along its path in a material, the nonlinear absorption is limited to only a small area in the beam focus. This, in consequence, allows for dense data writing with a 3D resolution [8]. The two-photon isomerisation process in azopolymers has been recently demonstrated using high peak-power 
ultra-short pulses [9-11]. The studies were performed for azo dyes dispersed in a flexible poly(methylmethacrylate) (PMMA) matrix. Such guest-host systems have an advantage in a relatively simple preparation and fast writing rates but, on the other hand, are characterised by a limited chromophore loading level and fast thermal relaxation of the induced order in dark.

Polyimide materials exhibit high glass transition temperature $\left(>200^{\circ} \mathrm{C}\right)$ and a rigid structure. It has been shown, however, that the photoinduced orientation in these materials occurs at room temperature $[12,13]$. Thus, polyimides having the azodyes covalently bonded to the main chain may offer a system of an increased stability of the written data.

This work is devoted to the photoinduced birefringence created in the azo-dye-functionalized polyimide forming a side-chain system. The investigated material was thermally stable with a decomposition temperature close to $400^{\circ} \mathrm{C}$ and amorphous [14], which is important when practical applications are considered. The birefringence was induced by high-intensity femtosecond pulses via a two-photon absorption process. The study presents the excitation intensity dependence of growths and decays of the material birefringence along with the obtained birefringence level and its temporal stability in dark.

\section{Experimental}

The synthesis of substrate materials for the investigated polyimide (PI-Az) was carried out according to the procedure presented in [15]. The synthesis and characterization of $\mathrm{PI}-\mathrm{Az}$ was described in [14].

The polymer films were obtained from a homogenous solution of PI-Az in NMP filtred through $0.2 \mu \mathrm{m}$ membranes and cast onto glass substrates. The films were dried at $200^{\circ} \mathrm{C}$ for 2 hours. The linear optical properties of PI-Az were analysed by UV-vis absorption spectroscopy using a JASCO V570 UV-V-NIR spectrometer. The UV-vis spectra were acquired both in polymer solution in NMP and in solid thin form on the glass substrate.

The sample birefringence was induced by the 100 -fs pulses at $800 \mathrm{~nm}$ and a $1-\mathrm{kHz}$ repetition rate delivered by a Ti:sapphire regenerative amplifier (Spitfire, Spectra Physics). The incident pulse energies were varied using an achromatic halfwave plate and a calcite polarizer. In order to obtain sufficient optical densities, the writing beam was focused using a spherical lens, and the polymer film was placed in front of the focal plane. A weak $690 \mathrm{~nm}$ in wavelength beam from a laser diode was used for probing the induced birefringence changes in the material. The beam was passing perpendicularly through the sample placed between two crossed polarizers and was centred at a writing beam spot (of $\sim 3$-mm diameter). For a maximum resulting change in the transmission signal, the polarization of the writing beam was set at $45^{\circ}$ with respect to the horizontal polarization direction of the probing beam. The transmission of the probe beam through crossed polarizers setup was detected by a lock-in amplifier using a mechanical chopper for modulation of the probing beam. The measurements were performed for the same sample point as a function of average writing power increased from a value of $50 \mathrm{~mW}$ to $700 \mathrm{~mW}$, which was the maximum power obtained from the laser system. The above optical powers corresponded to peak intensities of $7.1 \mathrm{GW} / \mathrm{cm}^{2}$ and $99.4 \mathrm{GW} / \mathrm{cm}^{2}$, respectively. After a 6-minute-irradiation cycle, the beam was switched off for 400 seconds. Then, an achromatic quarter wave plate was inserted into the writing beam way. The beam of a circular polarization and $150-\mathrm{mW}$ power was being switched on until the transmitted signal reached the value prior to first illumination cycle. For optical induced birefringence measurements, the polymer film of $4.8-\mu \mathrm{m}$ thickness measured by a stylus profiler was used.

\section{Results and discussion}

The chemical structure of the investigated photochromic polyimide is shown in Fig. 1.

The specific structure of PI-Az material is due to the fact that one of the aromatic azobenzene cores is a part of the backbone connected in the 2 and 4 positions with imide ring. The chromophore forms the molecule of an acceptor$\pi$-acceptor type.

Electronic spectrum of PI-Az solution in NMP exhibits two absorption bands (Fig. 2a). The one in the UV region
Fig. 1 Chemical structure of the investigated polyimide (PI-Az)

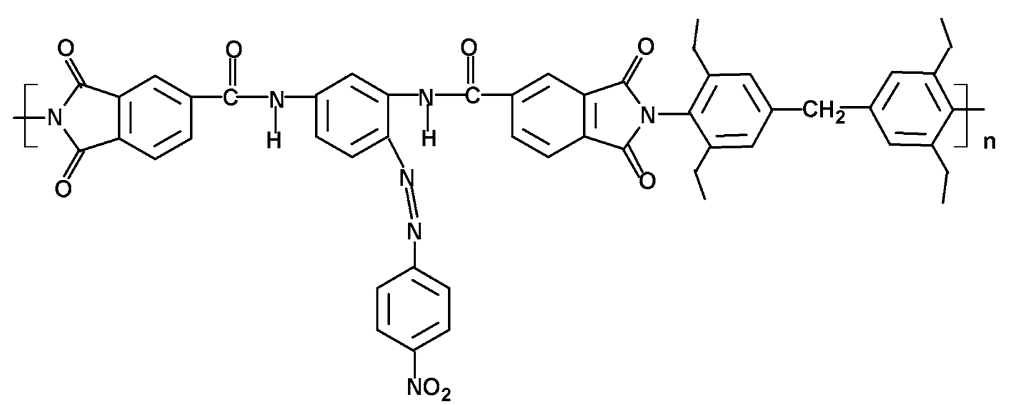




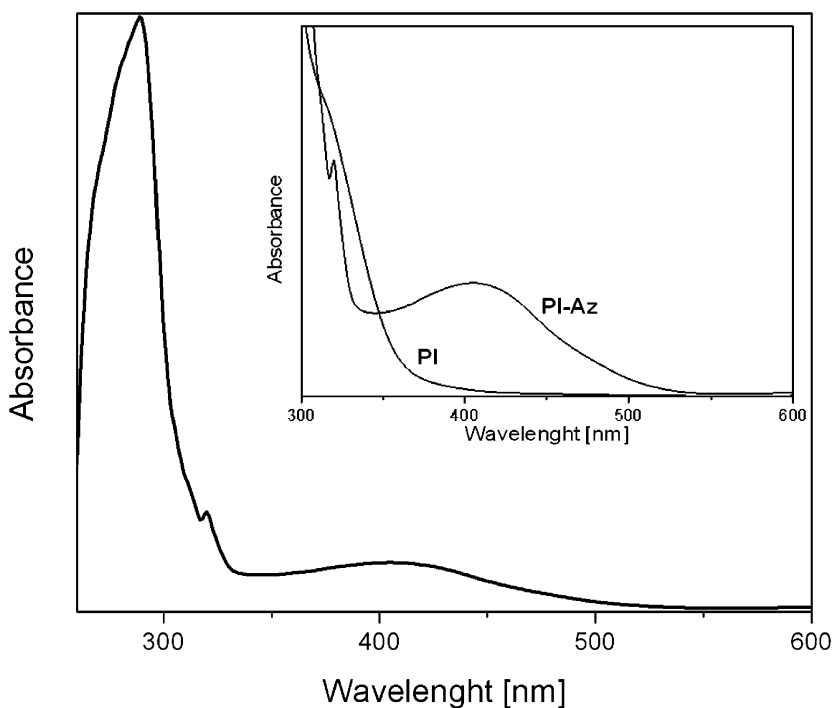

a

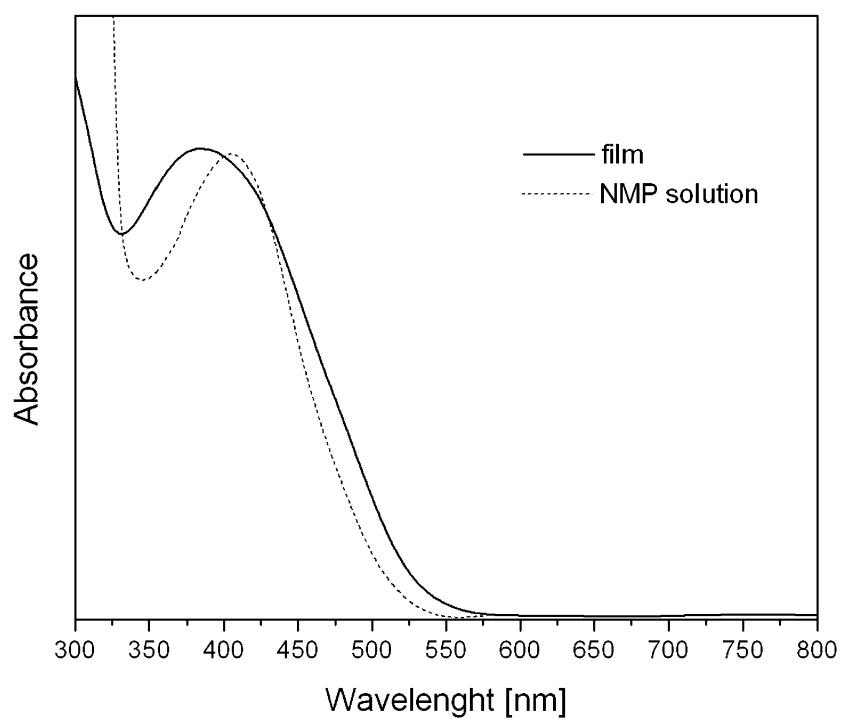

b

Fig. 2 UV-vis spectra of PI-Az in NMP solution compared with (a) PI spectrum in inset, (b) PI-Az film spectrum

centered at about $290 \mathrm{~nm}$ is attributed to transition within the polymer backbone. The second one, at around $404 \mathrm{~nm}$ is assigned to the electronic transition moment of the transazochromophore.

In order to confirm such an assignation of the absorption bands, the additional polyimide material without the azobenzene group (PI) was synthesised. Its UV-vis spectrum is compared with the spectrum of $\mathrm{PI}-\mathrm{Az}$ in the inset of Fig. 2a. As it can be seen from the figure in the case of the PI polymer, only the absorption band in UV region without absorption above $360 \mathrm{~nm}$ was found.

Absorption spectra of the polyimide in solution and film exhibited the same shape, but a slightly broader band was observed in the case of the film (Fig. 2b). A 16-nm shift

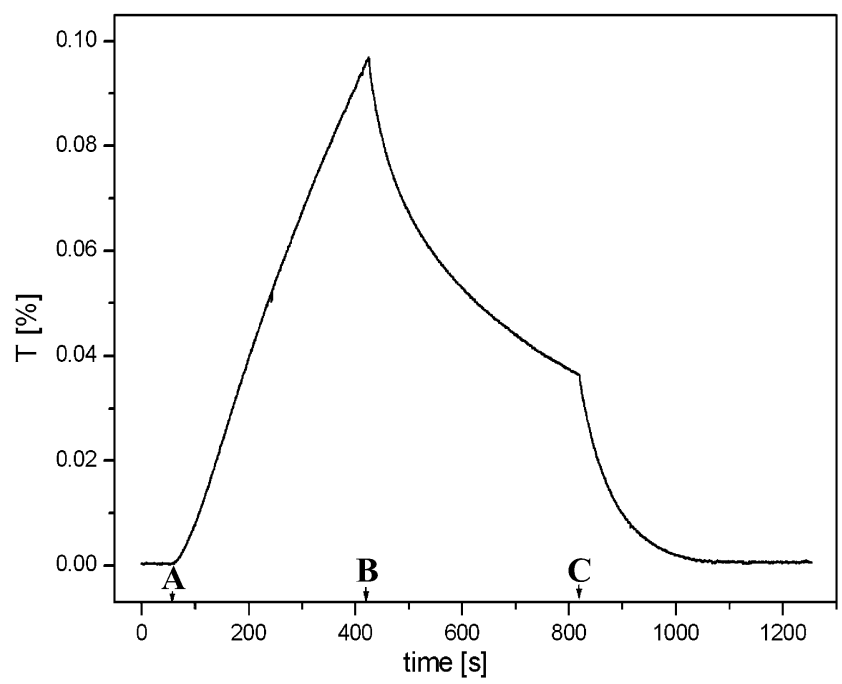

Fig. 3 Transmission curve representing writing, relaxation and erasing sequence for PI-Az film irradiated with $100 \mathrm{~mW}$ of average power

of the film absorption maximum towards shorter wavelengths in comparison to that in the solution indicates possible H-type aggregation of azobenzene chromophores in the film, similarly to what was observed for other azopolymers $[16,17]$. As it can be seen from the figure, the PI-Az polymer film is transparent at the probing and writing wavelengths. However, half of the writing wavelength falls close to the maximum of the absorption peak.

Figure 3 presents an exemplary transmission curve recorded for $100 \mathrm{~mW}$ of the average writing power.

Prior to the sample illumination with the writing light, zero transmission through the system was detected. This was as a consequence of a random chromophore orientation and isotropic properties of the polymeric material. Switching on the writing light (time A in Fig. 3) led to a monotonous increase in probe beam transmission signal, which was indicative of an induced birefringence. After switching off the writing beam (time B in Fig. 3) the signal started to go down due to a thermal randomization of the chromophore alignment. Subsequent sample illumination with the light of a circular polarization (time $\mathrm{C}$ in the figure) initiated the process of erasing the material birefringence. The erasing was complete in the cases where the birefringence was created by the writing light of the powers ranging from $50 \mathrm{~mW}$ to $400 \mathrm{~mW}$. In the case of $700 \mathrm{~mW}$ of the writing power the erasing was not complete; the transmission signal decreased to a final value, which gave a remnant sample birefringence of ca. 0.001 (see (1)). We may thus conclude that apart from the highest applied optical power the writing beam did not cause the material degradation. However, some structural changes might appear during the $700-\mathrm{mW}$ writing cycle. It was shown by Ishitobi et al. that the guest-host system of Disperse Red 1 dissolved in PMMA undergoes an irre- 


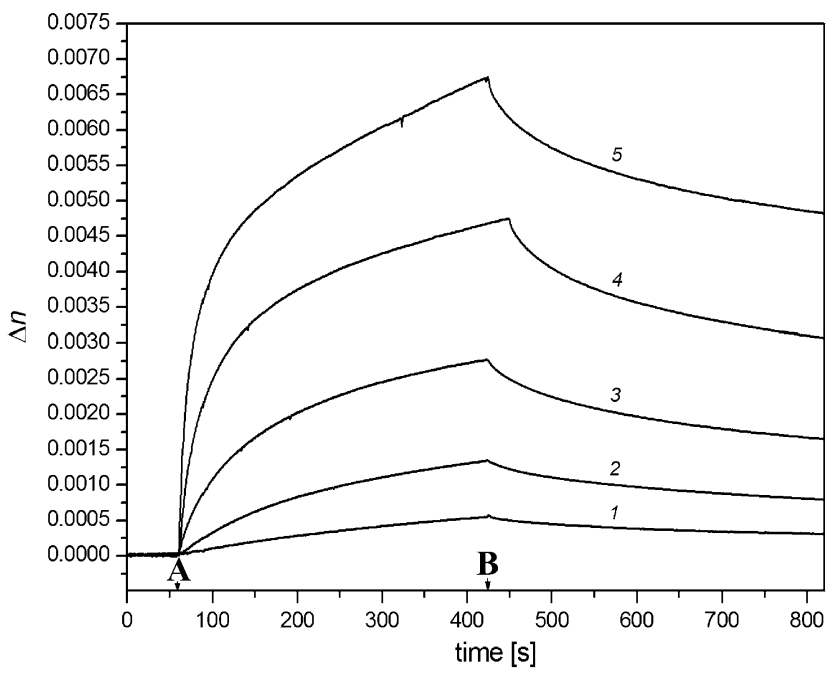

Fig. 4 Generation and relaxation curves of induced birefringence in $\mathrm{PI}-\mathrm{Az}$ film obtained for different writing powers. $1-50 \mathrm{~mW}$, 2-100 mW, 3-200 mW, 4-400 mW, 5-700 mW

versible photobleaching under the $785 \mathrm{~nm}$ irradiation via a multi-photon absorption process [18].

The measured transmittance through the crossed-polarizer set-up was used for the determination of the optically induced birefringence knowing that

$\Delta n=\frac{\lambda}{\pi d} \sin ^{-1} \sqrt{T}$,

where $\lambda$ is the probing beam wavelength, $d$ is the film thickness, $T$ is the transmittance of the setup, and $\Delta n$ is the difference in refractive indices for polarizations parallel and perpendicular to the polarization direction of the writing beam. Figure 4 shows the generation and relaxation of the induced birefringence in the studied polymer film for all applied irradiation powers. In order to verify that the observed birefringence was induced by two-photon absorption, the initial writing rates were analysed as functions of the average optical writing power. The logarithmic plot of $\frac{d \Delta n}{d t}$ versus $P_{a v}$ is shown in Fig. 5. The fitted line of a slope $(1.9 \pm 0.1)$ was obtained, what within the error is predicted for two-photon isomerization processes. It should be pointed out that such dependence was observed in the writing power range, which was essentially wider than the range applied by other groups $[11,19,20]$.

The birefringence growth and relaxation with time are often described by the biexponential equations

$$
\begin{aligned}
& \Delta n=A\left[1-\exp \left(-t / \tau_{A}\right)\right]+B\left[1-\exp \left(-t / \tau_{B}\right)\right], \\
& \Delta n=C \exp \left(-t / \tau_{c}\right)+D \exp \left(-t / \tau_{D}\right)+E
\end{aligned}
$$

where $\tau_{A}, \tau_{B}, \tau_{C}$, and $\tau_{D}$ are the time constants of processes appearing during writing or relaxation, respectively, $A, B$, $C$, and $D$ are the amplitudes associated with the processes, and $E$ is the residual birefringence.

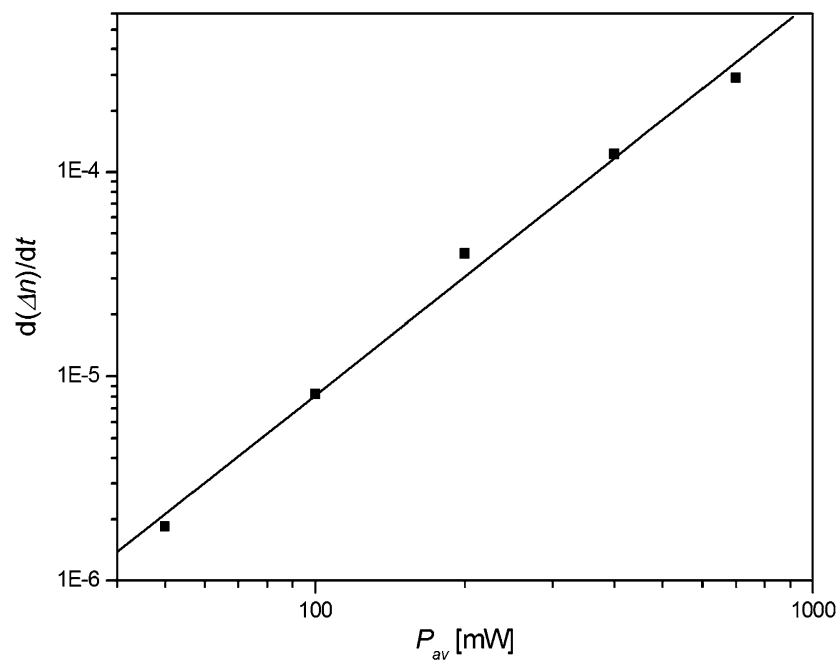

Fig. 5 The initial writing rates of the induced birefringence as function of the average writing power

It was found that in the investigated polyimide material the birefringence growths and decays could be well fitted using the above equations. However, the reliable set of fitted parameters for analysing the trends was obtained for three curves recorded during and after 200-, 400-, and 700-mW illumination.

The fitted parameters for (2) and (3) are presented in Table 1. The biexponential function (2) consists of two parts representing the fast and slow processes appearing during the writing cycle. As it can be seen from the table, the time constants of the fast process decrease with the writing power. The time constants of the slow process, in the case of $200 \mathrm{~mW}$ and $400 \mathrm{~mW}$ of the writing power, are almost equal and thus appear not to depend on the laser beam intensity. The $\tau_{B}$ time constant obtained for $700 \mathrm{~mW}$ is longer; however, as it was mentioned, at this power the laser light might induce some additional changes to the material that are not present under weaker light illumination conditions. What is interesting, for three examined optical powers, the contribution of the fast process to the final birefringence (represented by $A_{n}=A /(A+B)$ parameters $)$ is lower than that of the slow process (represented by $B_{n}$ ), and $A_{n}$ (and $B_{n}$ ) parameters are the same one in the case of applying 400 and $700 \mathrm{~mW}$.

The time constants $\tau_{C}, \tau_{D}$ and normalized parameters $C_{n}$ and $D_{n}$ (representing the contribution of both processes to the relaxation of birefringence) are close to each other, especially for $200 \mathrm{~mW}$ and $400 \mathrm{~mW}$ of the writing powers. This indicates that the rate of birefringence relaxation is independent on the writing laser power.

The biexponential kinetics of the writing process has been observed in the functionalized polymer systems containing the azobenzene molecules [5, 6, 21, 22]. However, the authors gave a different attribution of the fast and slow contributions. Ho et al. associated the fast process with the 
Table 1 Fitted parameters for (2) and (3). The subscript $n$ refers to normalized parameters, e.g. $A_{n}=A /(A+B)$; $C_{n}=C /(C+D+E)$

\begin{tabular}{llllllllll}
\hline$P_{a v}[\mathrm{~mW}]$ & $A_{n}$ & $B_{n}$ & $\tau_{A}[\mathrm{~s}]$ & $\tau_{B}[\mathrm{~s}]$ & $C_{n}$ & $D_{n}$ & $E_{n}$ & $\tau_{C}[\mathrm{~s}]$ & $\tau_{D}[\mathrm{~s}]$ \\
\hline 200 & 0.28 & 0.72 & 29 & 198 & 0.08 & 0.39 & 0.53 & 28 & 259 \\
400 & 0.45 & 0.55 & 20 & 195 & 0.09 & 0.33 & 0.58 & 29 & 270 \\
700 & 0.45 & 0.55 & 14 & 268 & 0.08 & 0.25 & 0.67 & 23 & 238 \\
\hline
\end{tabular}

reorientation of the azobenzene groups and the slow one with the aligning motion of the polymeric segments [21], while Song et al. related the fast and slow contributions to the formation of cis and trans isomers, respectively [5]. Attribution of the processes involved in the investigated polyimide during the writing cycle requires further studies. It should be noted, however, that both time constants associated with the birefringence growth are of the same order of magnitude comparing to the time constants observed by He et. al. for an azobenzene-containing fluorinated polyimide [23], while the time constants $\tau_{C}, \tau_{D}$ are longer than reported by $\mathrm{He}$ et al. The film birefringence obtained in this work in the case of $400-\mathrm{mW}$ irradiation is almost 0.005 . This is ca. two orders of magnitude more than the birefringence level reported for the system of Disperse Red 13 chromophore dispersed in PMMA matrix [11] and close to the value obtained by $\mathrm{He}$ et al. in polyimide material via the linear absorption process.

Similarly to He et al., the birefringence relaxation process is independent on the writing laser power. The birefringence decay is caused by the thermal cis-trans isomerization of azochromophores and a thermal randomization of the molecular orientation. We believe that the fast decay with time constant $\tau_{C}$ may be related to the former process, whereas the slow decay with time constant $\tau_{D}$ is due to the latter process. The thermal stability of the induced order is essentially higher than that in the methacrylate-based azopolymer [11]. After 6-minute monitoring the induced birefringence decreased by ca. $35 \%$ revealing a permanent data storage capacity. We attribute this result to a rigid functionalized polyimide structure.

\section{Conclusions}

The optical birefringence was obtained in azo-dye-functionalized polyimide material as a result of the chromophore reorientation accompanying the photoisomerization cycles. The birefringence was induced by intense femtosecond infrared pulses via two-photon absorption process. The kinetics of growth and decay of the induced birefringence were analysed as a function of the writing beam intensity. It was found that the detected growths and decays may be described by biexponential functions. The time constant associated with a fast process of birefringence growth decreased with the optical power, while the time constant associated with the slow process appeared not to depend on the writing power. Optical erasing of the birefringence was shown to proceed without causing the material damage apart from the case where the highest applied power was used for the writing cycle. High induced birefringence level and its dark stability were obtained.

Open Access This article is distributed under the terms of the Creative Commons Attribution Noncommercial License which permits any noncommercial use, distribution, and reproduction in any medium, provided the original author(s) and source are credited.

\section{References}

1. T. Todorov, L. Nikolova, N. Tomova, Appl. Opt. 23, 4309 (1984)

2. A. Natansohn, P. Rochon, J. Gosselin, S. Xie, Macromolecules 25, 2268 (1992)

3. R.H. Berg, S. Hvilsted, P.S. Ramanujam, Nature 383, 505 (1996)

4. A. Natansohn, P. Rochon, Chem. Rev. 102, 4139 (2002)

5. O.-K. Song, C.H. Wang, M.A. Pauley, Macromolecules 30, 6913 (1997)

6. M.J. Kim, J. Lee, C. Chun, D.Y. Kim, S. Higuchi, T. Nakayama, Macromol. Chem. Phys. 208, 1753 (2007)

7. V. Cimrova, D. Neher, R. Hildebrandt, M. Hegelich, A. Von der Lieth, G. Marowsky, R. Hagen, S. Kostromine, T. Bieringer, Appl. Phys. Lett. 81, 1228 (2002)

8. D.A. Parthenopoulos, P.M. Rentzepis, Science 245, 843 (1989)

9. H. Ishitobi, Z. Sekkat, S. Kawata, J. Chem. Phys. 125, 164718 (2006)

10. M. Maeda, H. Ishitobi, Z. Sekkat, S. Kawata, Appl. Phys. Lett. 85, 351 (2004)

11. C.R. Mendonca, U.M. Neves, L. De Boni, A.A. Andrade, D.S. dos Santos Jr., F.J. Pavinatto, S.C. Zilio, L. Misoguti, O.N. Oliveira Jr., Opt. Commun. 273, 435 (2007)

12. Z. Sekkat, J. Wood, E.F. Aust, W. Knoll, W. Volksen, R. Miller, J. Opt. Soc. Am. B 13, 1713 (1996)

13. E. Schab-Balcerzak, A. Sobolewska, A. Miniewicz, Opt. Mater. 31, 405 (2008)

14. D. Sęk, E. Schab-Balcerzak, E. Grabiec, Polymer 40, 2419 (1999)

15. D. Sęk, E. Grabiec, A. Miniewicz, A. Sobolewska, Proc. SPIE 5724, 311 (2005)

16. M.R.U. Cardoso, M. Neves, L. Misoguti, Zh. Ye, X.R. Bu, C.R. Mendonca, Opt. Mater. 28, 1118 (2006)

17. E. Schab-Balcerzak, M. Siwy, M. Kawalec, A. Sobolewska, A. Chamera, A. Miniewicz, J. Phys. Chem. A 113, 8765 (2009)

18. H. Ishitobi, Z. Sekkat, S. Kawata, J. Opt. Soc. Am. B 23, 868 (2006)

19. Z. Sekkat, H. Ishitobi, S. Kawata, Opt. Commun. 222, 269 (2003)

20. D.S. Correa, M.R. Cardoso, V.C. Goncalves, D.T. Balogh, L. De Boni, C.R. Mendonca, Polymer 49, 1562 (2008)

21. M.S. Ho, A. Natansohn, P. Rochon, Macromolecules 28, 6124 (1995)

22. M.V. Kozlovsky, V.V. Lazarev, Macromol. Chem. Phys. 204, 1226 (2003)

23. T.C. He, C.S. Wang, X. Pan, C.Z. Zhang, G.Y. Lu, Appl. Phys. B 94, 653 (2009) 